\title{
FUNDAMENTAL SOLUTIONS FOR DIFFERENTIAL EQUATIONS ASSOCIATED WITH THE NUMBER OPERATOR
}

BY

YUH-JIA LEE

\begin{abstract}
Let $(H, B)$ be an abstract Wiener space. If $u$ is a twice $H$-differentiable function on $B$ such that $D u(x) \in B^{*}$ and $D^{2} u(x)$ is of trace class, then we define $\mathscr{T} u(x)=-\Delta u(x)+(x, D u(x))$, where $\Delta u(x)=$ trace $_{H} D^{2} u(x)$ is the Laplacian and $(\cdot, \cdot)$ denotes the $B-B^{*}$ pairing. The closure $\bar{\Re}$ of $\Re$ is known as the number operator. In this paper, we investigate the existence, uniqueness and regularity of solutions for the following two types of equations: (1) $u_{t}=-\Re u$ (initial value problem) and (2) $\Re^{k} u=f(k>1)$. We show that the fundamental solutions of (1) and (2) exist in the sense of measures and we represent their solutions by integrals with respect to these measures.
\end{abstract}

1. Introduction. Let $(H, B)$ be an abstract Wiener space [1]. $H$ is a given real separable Hilbert space with norm $|\cdot|=\sqrt{\langle,\rangle}$ and $B$ is the completion of $H$ with respect to a weaker norm \|\| (a measurable norm in the sense of L. Gross [1]). The integration over $B$ is performed with respect to the Wiener measure $p_{t}$ which is generated by the Gauss cylinder set measure with variance $t>0$ on $H$. It is well known from the constructive quantum field theory that the Fock space (over $H$ ) $\mathscr{F}(H)$ can be identified with $L^{2}\left(p_{1}\right)$ (see [9], [13]). Under this identification, we can further identify the number (of particles) operator on $\mathscr{F}(H)$ with the infinitesimal generator $\bar{\Re}$ of the Ornstein-Uhlenbeck semigroup for the Brownian motion on $B$. When $H$ is finite dimensional, $\overline{\mathscr{T}}$ is the closure of $\Re=-\Delta+x \cdot \nabla$. However $\mathscr{F}(H)$ is usually constructed over an infinite dimensional $H$, so a suitable infinite dimensional expression of $\Re$ as a differential operator must be formulated.

A formulation of $\Re$ which will be used in this paper is defined as follows: For a real-valued function $f$ on $B$, we may regard $f$ as a function $g$ defined in a neighborhood of the origin of $H$ by defining $g(h)=f(x+h)$. If $g$ is $k$-times Fréchet differentiable at 0 , then we say that $f$ is $k$-times $H$-differentiable at $x$ and we denote the $k$-fold $H$-derivative at $x$ by $D^{k} f(x)$. If $D^{2} f(x)$ is of trace class, we then define the Laplacian $\Delta f(x)=\operatorname{trace}_{H}\left[D^{2} f(x)\right]$ [2]. Next, we interpret the term " $x \cdot \nabla f(x)$ " by $(x, D f(x))$ if $D f(x) \in B^{*}$, where $(\cdot, \cdot)$ denotes the $B$ - $B^{*}$ pairing. Then we define $\Re f(x)=-\operatorname{trace}_{H}\left[D^{2} f(x)\right]+(x, D f(x))$. The domain of $\Re$ contains the $C^{2}$-functions on $B$ (when $f$ is $C^{2}, D^{2} f(x)$ is necessarily of trace class (see [4, p. 81; Goodman's Theorem])). It is well known that $\Re$ is a selfadjoint operator on $L^{2}\left(p_{1}\right)$ and the Hermite cylinder polynomials form a complete set of eigenfunctions

Received by the editors August 14, 1980 and, in revised form, December 1, 1980.

1980 Mathematics Subject Classification. Primary 35K15, 35J30; Secondary 28A40, 60 J45.

Key words and phrases. Abstract Wiener space, Wiener measure, $\boldsymbol{H}$-differentiation, number operator, fundamental solution. 
for $\mathscr{x}$. Moreover, $\mathscr{N}$ extends to the generator $\overline{\mathscr{T}}$ of the Ornstein-Uhlenbeck semigroup (which is a contraction semigroup on $\left.L^{2}\left(p_{1}\right)[12]\right) . \bar{\Re}$ is known as the number operator.

In this paper we shall concern ourselves with the existence, uniqueness and regularity properties of the fundamental solution of the differential operator $\Re^{k}$ for $k \geqslant 1$. From the work of Gross [2] and Piech [10], [11], we see that the fundamental solutions of certain parabolic and elliptic second order equations are given by measures. For the case of higher order equations, less work has been done. By using the technique of the Fourier-Wiener transform, we have shown that for any exponential type analytic function $f$ with $\int_{B} f(x) p_{1}(d x)=0$, the solution of $\Re^{k} u(x)=f(x)$ exists uniquely (up to an addition of a constant) and it can also be represented by a series which is always convergent uniformly on bounded subsets of $B$ [7]. (One might wish to solve the equation $\mathscr{T}^{k} u=f$ by using the Hermite polynomial expansion. Since the Hermite polynomial expansion is convergent in $L^{2}$ and " $\mathcal{H} f$ " exists only in a weak sense [12], we would not obtain any knowledge

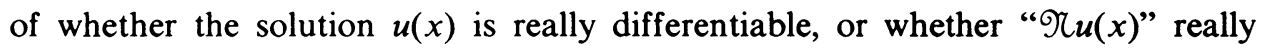
exists pointwise.) Following the approach of Gross [2], we shall show that there is a family of measures $\{Q(x, d y)\}$ (which serve as the "fundamental solution" of $\mathfrak{T}^{k}$ ) so that for a smooth function $f$ (possibly nonanalytic) with $\int_{B} f(x) p_{1}(d x)=0$, the function $Q f(x)=\int_{B} f(y) Q(x, d y)$ is, up to an addition of a constant, the only solution of $\mathfrak{x}^{k} u=f$. This also extends the results of [6, Theorem 5] and [7, Theorem 5.1].

In the course of proving the above result, we also obtain some regularity properties for the fundamental solution of the initial value problem associated with the equation $u_{t}(x, t)=-\Re u(x, t)$ and extend the results of Piech [11] to a class of unbounded initial functions.

The following lemma to be used later is an immediate consequence of [12].

Lemma 1.1. Assume $f$ and $g$ are in the domain of $\Re$. If $f, g,\|D f\|_{B^{*}},\|D g\|_{B^{*}}$, $\left\|D^{2} f\right\|_{\mathrm{tr}}$ and $\left\|D^{2} g\right\|_{\mathrm{tr}}$ (where \|\|$_{B^{*}}$ and \|\|$_{\mathrm{tr}}$ denote the $B^{*}$-norm and trace norm respectively) belong to $L^{2}\left(p_{1}\right)$, then we have

$$
\int_{B}(\Re f)(x) g(x) p_{1}(d x)=\int_{B} f(x)(\Re g)(x) p_{1}(d x)
$$

COROLlaRY 1.2. Let $g$ be an $L^{2}$ function. If $\Re u(x)=g(x)$ has a solution $u(x)$ in $L^{2}$ with either $\left\|D^{2} f(x)\right\|_{\mathrm{tr}}$ or $\|D f(x)\|_{B^{*}}$ being in $L^{2}\left(p_{1}\right)$, then we must have $\int_{B} g(x) p_{1}(d x)=0$.

REMARK 1.3. In this paper, all the functions are assumed to be real in order to avoid computational complexity. However, one can extend all the results to complex-valued functions in the natural way be considering the real and imaginary parts.

In what follows, we shall use " $D$ " or " $D_{x}$ " to denote the differentiation with respect to the space variable and use " $\partial / \partial t$ " or " $(\cdot)_{t}$ " to denote the derivative with respect to the time variable. 
2. Solution of $u_{t}=-\Re u$. In this section we shall consider the following Cauchy problem

$$
\begin{gathered}
\partial u(x, t) / \partial t=-\Re u(x, t) \quad(x \in B, t>0), \\
\lim _{t>0} u(x, t)=f(x) \quad \text { uniformly for } x \text { in bounded sets. }
\end{gathered}
$$

Our goal is to extend the results of Piech [11, Theorem 1] and Lee [7, Theorem 4.7] (both papers dealt with problem (2)) to a wider class of initial functions which includes both the class $Q$ of bounded uniformly Lip-1 functions and the class $\mathcal{E}_{a}(B)$ of real analytic exponential type functions.

The class of functions which shall be considered in this paper is defined as follows:

A real-valued measurable function $f$ is said to be in the class $\mathcal{L}$ if there are constants $c, c^{\prime} \geqslant 0$ such that

$$
|f(x)-f(y)| \leqslant c \cdot e^{c^{\prime}\|x\|} e^{c^{\prime}\|y\|}\|x-y\|
$$

for all $x, y$ in $B$ (see [8]).

It follows from Fernique's Theorem (see, for instance, [4]) that $\mathcal{Q}+\mathcal{E}_{a}(B) \subset \mathcal{L}$ $\subset L^{p}\left(p_{1}\right)(p \geqslant 1)$. Let $p_{t} f(x)=\int_{B} f(x+y) p_{t}(d y)$. If $f$ is in $\mathcal{L}$, then it is not hard to see that $p_{t} f$ is a $C_{H}^{1}$-function in $\mathcal{L}$. (By a $C_{H}^{1}$-function we mean an $H$-differentiable function $f$ defined on $B$ such that $D f(x)$ is continuous from $B$ into $H^{*}$ with the norm topology.)

We shall establish the following:

THEOREM 2.1. Assume that $f$ is a function in L. Let

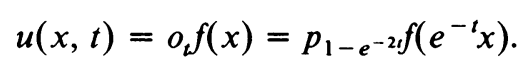

Then we have

(a) $D u(x, t) \in B^{*}$ and $\|D u(x, t)\|_{B^{*}} \leqslant c_{1} e^{-t} e^{2 c^{\prime}\|x\|}$ for some constants $c_{1}$, $c^{\prime} d e-$ pending only on $f$.

(b) For each $t_{1}, t_{2}>0$ and any bounded set $U$, the map $(x, t) \rightarrow D^{2} u(x, t)$ is uniformly continuous on $U \times\left[t_{1}, t_{2}\right]$ into the Banach space of trace class operators on $H$.

(c) $\left\|D^{2} u(x, t)\right\|_{\mathrm{tr}} \leqslant c_{2} t^{-1 / 2} e^{-t} e^{c^{\prime}\|x\|}$ for some constants $c_{2}, c^{\prime}$ which depend only on $f$.

(d) The function $u(x, t)=o_{t} f(x)$ is jointly uniformly continuous on $U \times[0, \lambda]$ for each finite $\lambda>0$ and each bounded subset $U$ of $B$.

(e) For each $t>0,(\partial / \partial t) u(x, t)$ exists and $(\partial / \partial t) u(x, t)=-\Im u(x, t)$. Moreover, $\lim _{t \backslash 0} u(x, t)=f(x)$ uniformly in $U$.

The proof of Theorem 2.1 will be accomplished by the following lemmas.

Lemma 2.2 [8]. Assume that $f \in \mathcal{L}$ and $v(x, t)=p_{t} f(x)$. Then the function $x \rightarrow$ $p_{t} f(x)$ is infinitely $H$-differentiable. Moreover, we have

(a) $D^{2} v(x, t)$ is a trace class operator for each $x$ in $B$ and $t>0$. Furthermore,

$$
\left\|D^{2} v(x, t)\right\|_{\mathrm{tr}} \leqslant c_{f} \cdot t^{-1 / 2} e^{c^{\prime}\|x\|}\left\{\int_{B} e^{2 c^{\prime}\|y\|} p_{t / 2}(d y)\right\}^{3 / 2},
$$

where the constants $c_{f}$ and $c^{\prime}$ depend only on $f$. 
(b) For each pair of positive numbers $a, a^{\prime}$ and each bounded set $U$, the map $(x, t) \rightarrow D^{2} v(x, t)$ is uniformly continuous on $U \times\left[a, a^{\prime}\right]$ into the Banach space of trace class operators on $H$.

(c) For each $t>0, \partial v / \partial t$ exists uniformly for $x$ in a bounded set and $(\partial / \partial t) v(x, t)$ $=\frac{1}{2} \operatorname{trace}_{H}\left[D^{2} v(x, t)\right]$. Moreover, $\lim _{t \backslash 0} v(x, t)=f(x)$ uniformly for $x$ in each bounded set.

The next lemma is useful for proving Theorem 2.1. Since its proof is quite similar to that of [11, Lemma 2.5] or [8, Lemma 1], we omit the proof.

LemMa 2.3 (SEE [11]). Assume $f$ is a function in $\mathcal{L}$ satisfying the condition (3). Then we have

(a) $D p_{t} f(x) \in B^{*}$ and $\left\|D p_{t} f(x)\right\|_{B^{*}} \leqslant c \cdot\left(\int_{B} e^{2 c^{\prime}\|y\|} p_{t}(d y)\right) e^{2 c^{\prime}\|x\|}$.

(b) The map $(y, x) \rightarrow\left(y, D p_{t} f(x)\right)$ is continuous.

(c) For fixed $t$ and $x$,

$$
\lim _{s \succ 0} s^{-1}\left(p_{t} f(x+s y)-p_{t} f(x)\right)=\left(y, D p_{t} f(x)\right)
$$

for all $y$ in B. Moreover, for fixed $t$ and $y$, the limit exists uniformly for $x$ in $a$ bounded set.

REMARK 2.4. In general, $p_{t} f(x)$ is not Fréchet differentiable in $B$. However it follows from Lemma 2.3 that $p_{t} f(x)(f \in \mathcal{L})$ has directional derivatives in all directions of $B$.

Proof OF TheOREM 2.1. Let $a(t)=1-e^{-2 t}$ and $b(t)=e^{-t}$. Then $u(x, t)=$ $p_{a(t)} f(b(t) x)$.

(a) Since $f\left(e^{-t} x\right)$ is in $\mathcal{E}$, Lemma 2.3 ensures that $D u(x, t)$ belongs to $B^{*}$ and $D u(x, t)=D_{x} p_{a(t)} f(b(t) x)=\left.e^{-t} D_{y} p_{a(t)} f(y)\right|_{y=b(t) x}$. Also by 2.3(a) we have

$$
\begin{aligned}
\|D u(x, t)\|_{B^{*}} & \leqslant c\left(\int_{B} e^{2 c^{\prime}\|y\|(\sqrt{a(t)})} p_{1}(d y)\right) e^{-t} e^{2 c^{\prime}\|x\|} \\
& \leqslant c\left(\int_{B} e^{2 c^{\prime}\|y\|} p_{1}(d y)\right) e^{-t} e^{2 c^{\prime}\|x\|} \\
& =c_{1} e^{-t} e^{2 c^{\prime}\|x\|} .
\end{aligned}
$$

(b) Since the map $\left.(x, t) \rightarrow D_{y}^{2} p_{a(t)} f(y)\right|_{y=e^{-t} x}$ is the composition of the following two uniformly continuous functions $(x, t) \rightarrow\left(e^{-t} x, a(t)\right)$ and $(x, t) \rightarrow D^{2} p t f(x)$, (b) follows from Lemma 2.2(b) and the fact $D^{2} u(x, t)=\left.e^{-2 t} D_{y}^{2} p_{a} f(y)\right|_{y=b x}$.

(c) By Lemma 2.2(a), we have

$$
\left\|D^{2} p_{t} f(x)\right\|_{\mathrm{tr}} \leqslant c_{f} \cdot\left(\int_{B} e^{2 c^{\prime}\|y\|} p_{1}(d y)\right)^{3 / 2} t^{-1 / 2} e^{c^{\prime}\|x\|} .
$$

It follows that

$$
\begin{aligned}
\left\|D^{2} u(x, t)\right\|_{\mathrm{tr}} & \leqslant c_{f} \cdot\left(\int_{B} e^{2 c^{\prime}\|y\|} p_{1}(d y)\right)^{3 / 2} e^{-2 t}\left(1-e^{-2 t}\right)^{-1 / 2} e^{c^{\prime}\|x\|} \\
& \leqslant c_{1} t^{-1 / 2} e^{-t} e^{c^{\prime}\|x\|},
\end{aligned}
$$

where $c_{1}=2 \cdot c_{f} \cdot\left(\int_{B} e^{2 c^{\prime}\|y\|} p_{1}(d y)\right)^{3 / 2}$ which depends only on $f$. 
(d) Let $F(x, t)=(b(t) x, a(t))$ and $v(x, t)=p_{t} f(x) . F(x, t)$ is uniformly continuous on $U \times[0, \lambda]$ and $u(x, t)=v \cdot F(x, t)$. Observe that if $\|x\| \leqslant M$ in $U$ the range of $F$ is a subset of $\{\|x\| \leqslant M\} \times[0,1]$ on which $v(x, t)$ is also uniformly continuous. Therefore $u(x, t)$, as a composition of two uniformly continuous functions, is uniformly continuous.

(e) It follows by the chain rule that $\partial u / \partial t$ exists and

$$
(\partial / \partial t) u(x, t)=(\partial / \partial a) p_{a} f(b x)(\partial a / \partial t)+(\partial / \partial b) p_{a} f(b x)(\partial b / \partial t) .
$$

Next, by (c) of Lemma 2.2, we see that

$$
\begin{aligned}
\left.(\partial / \partial a) p_{a} f(y)\right|_{y=b x} & =\left.\frac{1}{2} \operatorname{trace}_{H}\left[D^{2} p_{a} f(y)\right]\right|_{y=b x} \\
& =\frac{1}{2} e^{2 t} \operatorname{trace}_{H}\left[D_{x}^{2} p_{a} f(b x)\right]=\frac{1}{2} e^{2 t} \operatorname{trace}_{H}\left[D^{2} u(x, t)\right] .
\end{aligned}
$$

Similarly,

$$
\begin{aligned}
(\partial / \partial b) p_{a} f(b x) & =\left.\left(x, D p_{a} f(y)\right)\right|_{y=b x}=e^{t}\left(x, D_{x} p_{a} f(b x)\right) \\
& =e^{t}(x, D u(x, t)) .
\end{aligned}
$$

Putting (5) and (6) into (4), we get

$$
(\partial / \partial t) u(x, t)=\operatorname{trace}_{H}\left[D^{2} u(x, t)\right]-(x, D u(x, t))=-\Re u(x, t) .
$$

Finally,

$$
\begin{aligned}
|u(x, t)-f(x)| & =\left|\int_{B} f\left(e^{-t} x+\left(1-e^{-2 t}\right)^{1 / 2} y\right) p_{1}(d y)-f(x)\right| \\
& \leqslant \int_{B}\left|f\left(e^{-t} x+\left(1-e^{-2 t}\right)^{1 / 2} y\right)-f(x)\right| p_{1}(d y) \\
& \leqslant c \cdot e^{2 c^{\prime}\|x\|}\left\{\left(1-e^{-2 t}\right)^{1 / 2}\left(\int_{B} e^{c^{\prime}\|y\|}\|y\| p_{1}(d y)\right)\right. \\
& \rightarrow 0 \quad \text { uniformly on bounded subsets of } B
\end{aligned}
$$

as $t$ goes to 0 . This completes the proof of Theorem 2.1.

REMARK 2.5. If we define $o_{t}(x, A)=p_{1-e^{-2 t}}\left(e^{-t} x, A\right)$ for each $x$ in $B$ and for each Borel set $A$, then $o_{t}(x, \cdot)$ is a probability measure and the family $\left\{o_{t}(x, d y)\right\}$ serves as the fundamental solution for the Cauchy problem (2).

As a further application of Lemma 1.1, we prove the uniqueness for the Cauchy problem (2) in the following.

TheOrem 2.6 (UNIQUeness). Assume that $u(x, t)$ is a function defined on $B \times$ $[0, \infty)$ satisfying the following conditions:

(1) $u(x, t)$ is jointly continuous on $B \times[0, \infty)$ and, for each $s>0, u(x, t) \in$ $L^{2}(B \times[0, s])$.

(2) $u(\cdot, t)$ is twice $H$-differentiable such that $D u(x, t) \in B^{*}$ and $D^{2} u(x, t)$ is of trace class. Moreover, $\|D u(x, t)\|_{B^{*}}$ and $\left\|D^{2} u(x, t)\right\|_{\mathrm{tr}}$ are in $L^{2}\left(p_{1}\right)$ and, for each $s>0, u(x, t) \in L^{2}(B \times[0, s])$.

(3) $\partial u / \partial t$ exists and $(\partial / \partial t) u(x, t)=-\Re u(x, t)$.

(4) $\lim _{t>0} u(x, t)=0$.

Then $u(x, t)=0$ (see also [11]). 
Proof. Note that condition (1) implies that, for almost all $s \geqslant 0, u(\cdot, s) \in$ $L^{2}\left(p_{1}\right)$. Let $N$ be the exceptional set and $s \in(0, \infty) \backslash N$. For any $f \in \mathcal{E}_{a}(B)$, we have

$$
\int_{0}^{s} \int_{B} \Re u(x, t) o_{s-t} f(x) p_{1}(x) d t=\int_{0}^{s} \int_{B} u(x, t) \Re o_{s-t} f(x) p_{1}(d x) d t
$$

(by $2.6(2), 2.1$ and 1.1)

$$
\begin{aligned}
= & \int_{B} \int_{0}^{s} u(x, t) \frac{\partial}{\partial t} o_{s-t} f(x) d t p_{1}(d x) \\
= & \int_{B} \lim _{t \rightarrow s} u(x, t) o_{s-t} f(x)-\lim _{t \rightarrow 0} u(x, t) o_{s-t} f(x) \\
& -\int_{0}^{s} \frac{\partial u}{\partial t}(x, t) \cdot o_{s-t} f(x) d t p_{1}(d x) \\
= & \int_{B} u(x, s) f(x) p_{1}(d x)+\int_{B} \int_{0}^{s} \Re u(x, t) o_{s-t} f(x) p_{1}(d x) .
\end{aligned}
$$

This implies that $\int_{B} u(x, s) f(x) p_{1}(d x)=0$ for arbitrary $f$ in $\varepsilon_{a}(B)$. Since $u(\cdot, s) \in$ $L^{2}\left(p_{1}\right)$ and $\mathcal{E}_{a}(B)$ is dense in $L^{2}\left(p_{1}\right)$, hence $u(\cdot, s)=0$ a.e. $\left(p_{1}\right)$. Since $u(\cdot, s)$ is continuous, $u(\cdot, s)=0$. Thus we have proved that, for almost all $s>0, u(\cdot, s)=0$. Finally, by the continuity of $u(x, \cdot)$, we conclude that $u(x, s)=0$ for all $x$ and $s$.

It follows from Theorem 2.1 that for any $f$ in $\mathcal{L}, o_{\downarrow} f(x)$ always satisfies conditions (1)-(3) of Theorem 2.6. Thus we have the following

Corollary 2.7 (UniQueness). Assume that $u(x, t)$ is a function defined on $B \times[0, \infty)$ satisfying conditions (1)-(3) of Theorem 2.6. Suppose that $f$ is in the class $\mathcal{L}$ and $\lim _{t \rightarrow 0} u(x, t)=f(x)$. Then $u(x, t)=o_{\downarrow} f(x)$.

3. Solution of $\Re^{k} u=f$. In this section, we shall show that there exists a family of measures $\{Q(x, d y)\}$ such that for $f \in \mathcal{E}$ with mean zero, the function $u(x)=$ $\int_{B} f(y) Q(x, d y)$ solves the equation $\Re^{k} u=f$. This extends the result of [7, Theorem 5.1] and when $k=1$, this also extends [6, Theorem 5]. Furthermore, we shall show that the solution is unique.

Let $o_{t}(x, A)=p_{1-e^{-2 t}}\left(e^{-t} x, A\right)$ and $G(x, A)=\int_{0}^{\infty} o_{t}(x, A) d t$, where $x \in B$ and $A$ is a Borel set in $B$.

Obviously, $G(x, \cdot)$ is a Borel measure.

Let $o_{t} f(x)=\int_{B} f(y) o_{t}(x, d y)$ as in the previous section and define

$$
G f(x)=\int_{0}^{\infty} o_{t} f(x) d t \text { (if it exists) }
$$

Notation. We shall denote by $\varrho_{0}$ the subclass of functions $f$ in $\mathcal{L}$ with $\int_{B} f(x) p_{1}(d x)=0$.

The following proposition is basic.

Proposition 3.1. Assume $f \in \mathscr{L}_{0}$; then the integral $\int_{0}^{\infty} o_{t} f(x) d t$ is convergent and Gf also belongs to $\varrho_{0}$. Thus $G$, as a mapping, maps $\mathcal{L}_{0}$ into $\mathcal{L}_{0}$. 
Proof. Since $f$ is of mean zero, we may write

$$
o_{t} f(x)=\int_{B} f\left(e^{-t} x+\left(1-e^{-2 t}\right)^{1 / 2} y\right) p_{1}(d y)-\int_{B} f(y) p_{1}(d y) .
$$

Suppose that $f$ satisfies the inequality (3) (see the definition of $\mathscr{L}$ ). We estimate

$$
\begin{aligned}
\left|o_{t} f(x)\right| & \leqslant \int_{B}\left|f\left(e^{-t} x+\left(1-e^{-2 t}\right)^{1 / 2} y\right)-f(y)\right| p_{1}(d y) \\
& \leqslant \int_{B} c \cdot e^{c^{\prime}\|x\|} e^{2 c^{\prime}\|y\|}\left[e^{-t}\|x\|+\left|\left(1-e^{-2 t}\right)^{1 / 2}-1\right|\|y\|\right] p_{1}(d y) \\
& \leqslant c_{1} e^{c^{\prime}\|x\|}\|x\| e^{-t}+c_{2} e^{c^{\prime}\|x\|} e^{-2 t} \\
& \leqslant c_{3} e^{c^{\prime \prime}\|x\|}\|x\|\left(e^{-t}+e^{-2 t}\right),
\end{aligned}
$$

where $c^{\prime \prime}=c^{\prime}+1$ and $c_{3}=c \cdot \int_{B} e^{2 c^{\prime}\|y\|}(1+\|y\|) p_{1}(d y)$. Consequently,

$$
\int_{0}^{\infty}\left|o_{t} f(x)\right| d t \leqslant(3 / 2) \cdot c_{3} e^{c^{\prime \prime}\|x\|}=c_{4} e^{c^{\prime \prime}\|x\|}
$$

Next, by Fubini's Theorem and the semigroup property of $p_{t}$, we see that

$$
\begin{aligned}
\int_{B} G f(x) p_{1}(d x) & =\int_{0}^{\infty} \int_{B} \int_{B} f(x+y) p_{e^{-2 t}}(d x) p_{1-e^{-2 t}}(d y) d t \\
& =\int_{0}^{\infty}\left[\int_{B} f(x) p_{1}(d x)\right] d t=0 .
\end{aligned}
$$

Finally, for $x, y$ in $B$,

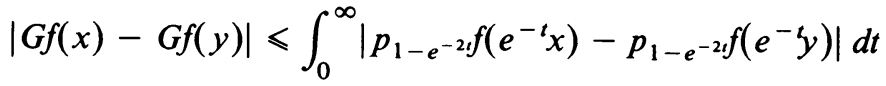

$$
\begin{aligned}
& \leqslant c\left\{\int_{B} e^{2 c^{\prime}\|z\|} p_{1}(d z)\right\}\left\{\int_{0}^{\infty} e^{-t} d t\right\} e^{c^{\prime}\|x\|} e^{c^{\prime}\|y\|}\|x-y\| \\
& \leqslant c_{1} e^{c^{\prime}\|x\|} e^{c^{\prime}\|y\|}\|x-y\| \text {. }
\end{aligned}
$$

Thus $G f \in \mathfrak{L}_{0}$.

Lemma 3.2. Assume $f \in \mathcal{L}_{0}$; then $\int_{0}^{\infty}\left|D o_{t} f(x)\right|_{H} d t$ and $\int_{0}^{\infty}\left|D^{2} o_{t} f(x)\right|_{H, H} d t$ are convergent. Moreover, for $h, k \in H$,

$$
\langle h, D G f(x)\rangle=\int_{0}^{\infty}\left\langle h, D o_{t} f(x)\right\rangle d t
$$

and

$$
\left\langle k, D^{2} G f(x) h\right\rangle=\int_{0}^{\infty}\left\langle k, D^{2} o_{\imath} f(x) h\right\rangle d t
$$

Proof. Since the $B^{*}$-norm $\|\cdot\|_{B^{*}}$ is stronger than the $H$-norm $|\cdot|_{H}$ and the trace norm $\|\cdot\|_{\mathrm{tr}}$ is stronger than the operator norm $|\cdot|_{H, H}$, the first assertion follows by (a) and (c) of Theorem 2.1. The second assertion follows from (b) of Theorem 2.1 and Lemma 2.3. 
Proposition 3.3. Assume $f$ is an $L^{2}\left(p_{1}\right)$ function.

(i) In order that there exists a $u(x) \in \mathcal{L}$ such that $\mathscr{T} u(x)$ exists and that $\mathcal{T} u(x)=f(x)$, it is necessary that $\int_{B} f(x) p_{1}(d x)=0$.

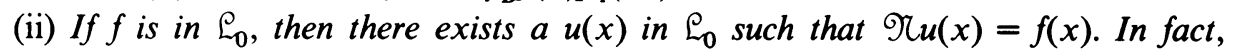
$\Re(x)=f(x)$.

Proof. (i) is a consequence of Corollary 1.2 and the fact that $\|D u(x)\|_{B^{*}} \leqslant c$. $e^{2 c^{\prime}\|x\|}$ which belongs to $L^{2}\left(p_{1}\right)$.

It remains to prove (ii). By (8) (Lemma 3.2), we have

$$
\begin{aligned}
\left|\int_{0}^{\infty}\left\langle h, D o_{t} f(x)\right\rangle d t\right| & \leqslant\left|\int_{0}^{\infty}\left(h, D o_{\imath} f(x)\right) d t\right| \\
& \leqslant\|h\| \int_{0}^{\infty}\left\|D o_{t} f(x)\right\|_{B^{*}} d t \quad \text { (by 2.1) } \\
& \leqslant\|h\| \cdot c_{1} \cdot e^{2 c^{\prime}\|x\|}<\infty .
\end{aligned}
$$

It follows that $|\langle h, D G f(x)\rangle| \leqslant c_{1} \cdot e^{2 c^{\prime}\|x\|}\|h\|$ and so $D G f(x) \in B^{*}$ and $\|D G f(x)\|_{B^{*}}$ $\leqslant c_{1} \cdot e^{2 c^{\prime}\|x\|}$.

Next, by (9) (Lemma 3.2), we have

$$
\left\langle k, D^{2} G f(x) h\right\rangle=\int_{0}^{\infty}\left\langle k, D^{2} o_{t} f(x) h\right\rangle d t \quad \text { for all } h, k \text { in } H .
$$

Now for any compact operator $T$ on $H$ and for any orthonormal basis $\left\{e_{j}\right\}$ in $H$, we see that

$$
\begin{aligned}
\left|\operatorname{trace}_{H}\left(T D^{2} G f(x)\right)\right| & =\left|\sum_{j=1}^{\infty}\left\langle T^{*} e_{j}, D^{2} G f(x) e_{j}\right\rangle\right| \\
& \leqslant \sum_{j=1}^{\infty} \int_{0}^{\infty}\left|\left\langle T^{*} e_{j}, D^{2} o_{\imath} f(x) e_{j}\right\rangle\right| d t \\
& \leqslant \int_{0}^{\infty}\left\|T D^{2} o_{t} f(x)\right\|_{\mathrm{tr}} d t \\
& \leqslant|T|_{H, H^{c_{2}}} e^{c^{\prime}\|x\|} \int_{0}^{\infty} t^{-1 / 2} e^{-t} d t \\
& <\infty \text { for each } x \text { in } B .
\end{aligned}
$$

Thus $D^{2} G f(x)$ is of trace class and $\left\|D^{2} G f(x)\right\|_{\mathrm{tr}} \leqslant c_{2} e^{c^{\prime}\|x\|}$. Therefore $G f(x)$ is in the domain of $\Re$.

Now, by Theorem 2.1, we get

$$
\begin{aligned}
\mathscr{N} G f(x) & =\int_{0}^{\infty} \Re o_{t} f(x) d t \\
& =\lim _{\varepsilon \rightarrow 0, r \rightarrow \infty}-\int_{\varepsilon}^{r} \frac{\partial}{\partial t} o_{t} f(x) d t=\lim _{\varepsilon \rightarrow 0, r \rightarrow \infty}\left(o_{B} f(x)-o_{r} f(x)\right) \\
& =f(x)-\int_{B} f(y) p_{1}(d y)=f(x) .
\end{aligned}
$$

This completes the proof of Proposition 3.3.

RemarK 3.4. Proposition 3.3 has shown that the family of measures $\{G(x, d y)\}$ forms a "fundamental solution" of the operator $\Re$. 
Notations. For $k \geqslant 1$, we shall use $\mathcal{L}(k)$ to denote the class of functions $g$ such that $\Re^{j} g$ exists for $j=0, \ldots, k-1\left(\Re^{0} g=g\right)$ and $\Re^{j} g \in \mathcal{L}$. We shall use $\mathcal{L}(k)_{0}$ to denote the subclass of functions $f$ in $\mathcal{L}(k)$ with $\int_{B} f(x) p_{1}(d x)=0$.

We let $G^{k} f(x)=G \circ G \circ \cdots(k$ times) $\cdots \circ G f(x)$ (if it exists) and define

$$
Q(x, A)=\int_{B} \int_{\substack{B \\ k-1 \text { times }}} \cdots \int_{B} \int_{B} G\left(y_{k-1}, A\right) G\left(y_{k-2}, d y_{k-1}\right) \cdots G\left(x, d y_{1}\right),
$$

where $x$ is in $B$ and $A$ is a Borel set in $B$.

Now if we consider the following system of equations

$$
\left\{\begin{array}{rll}
\Re u_{1}(x) & = & f(x), \\
\Re u_{2}(x) & = & u_{1}(x), \\
& \vdots & \\
\Re u_{k}(x) & = & u_{k-1}(x)
\end{array}\right.
$$

and then apply Proposition 3.1 and Proposition 3.3 to the system (11) repeatedly, we obtain the following

TheOREM 3.5. (a) Assume $f$ is an $L^{2}\left(p_{1}\right)$ function. Suppose that there exists a function $u(x)$ in the class $\mathcal{L}(k)$ such that $\Re^{k} u(x)$ exists and $\Re^{k} u(x)=f(x)$. We must have $\int_{B} f(x) p_{1}(d x)=0$.

(b) Assume $f$ is in the class $\varrho_{0}$ and let $Q(x, A)$ be the measure defined by (10). Then $G^{k} f(x)=\int_{B} f(y) Q(x, d y)$ exists, $G^{k} f \in \mathcal{L}(k)_{0}$ and $\Re^{k}\left(G^{k} f\right)(x)=f(x)$.

In particular, we have the following

Corollary 3.6 (SEe [6], [7]). Assume $f$ is a function in the class $\operatorname{Lip}_{1}+\mathcal{E}_{a}(B)$ with $\int_{B} f(x) p_{1}(d x)=0$. Then the function $u(x)=\int_{B} f(y) Q(x, d y)$, where $Q(x, d y)$ is defined by (10), solves the equation $\mathfrak{T}^{k} u(x)=f(x)$.

Remark 3.7. Let $\mathfrak{K}_{c} u(x)=-\Delta u(x)+c(x, D u(x)), c>0$. Then $\Re_{c}$ becomes a selfadjoint operator on $L^{2}\left(p_{c^{-1}}\right)$. After some suitable changes, for example: use " $\int_{B} f(x) p_{c^{-1}}(d x)=0$ " to replace " $\int_{B} f(x) p_{1}(d x)=0$ " and replace $o_{t}(x, d y)$ by the measure $r_{t}(x, d y)=p_{1-e^{-2 c t}}\left(e^{-c t} c^{1 / 2} x, d y\right)$, etc., one can easily verify that the results we have are still true if we replace $\Re$ by $\Re_{c}$.

We discuss the uniqueness of solutions in the following

REMARK 3.8. Obviously, the solution of $\Re^{k} u=f$ is not unique. For example, if $u$ is a solution and $C$ is a constant then $u+C$ is also a solution. However, it follows immediately from the $L^{2}$-theory of $\Re$ (see [12]) that if $u \in \mathcal{L}(k)$ and $\Re^{k} u=0$, then $u(x)=$ a constant. This implies that the solution of $\Re^{k} u=f, f \in \mathscr{\varrho}_{0}$, is of the form $u(x)=\int_{B} f(x) Q(x, d y)+C$ provided $u(x)$ is in the class $\mathcal{E}(k)$. (It is easy to see that the uniqueness of solutions still holds if we replace $\mathcal{L}(k)$ by the class $\delta(k)$, where $\mathcal{S}(k)=\left\{f \in L^{2}\left(p_{1}\right): f \in \operatorname{Domain}\left(\Re^{k}\right)\right.$ and $\left\|D\left(\Re^{j} f\right)(x)\right\|_{B^{*}} \in L^{2}$, for $j=$ $0,1, \ldots, k-1$, where $\left.\mathfrak{\Re}^{0} f=f\right\}$.) 


\section{REFERENCES}

1. L. Gross, Abstract Wiener spaces, Proc. 5th Berkeley Sympos. Math. Statist. Prob., vol. 2, Univ. California Press, Berkeley, 1965, pp. 31-42.

2. __ Potential theory on Hilbert space, J. Funct. Anal. 1 (1967), 123-181.

3. H.-H. Kuo, Integration by parts for abstract Wiener measures, Duke Math. J. 41 (1974), 373-379.

4. __ Gaussian measure in Banach spaces, Lecture Notes in Math., vol. 463, Springer-Verlag, Berlin and New York, 1975.

5. Distribution theory on Banach spaces, Probability in Banach Spaces, Lecture Notes in Math., vol. 576, Springer-Verlag, Berlin and New York, 1976.

6. __ Potential theory associated with Uhlenbeck-Ornstein process, J. Funct. Anal. 21 (1976), $63-75$.

7. Y.-J. Lee, Applications of the Fourier-Wiener transform to differential equations on infinite dimensional spaces. I, Trans. Amer. Math. Soc. 262 (1980), 259-283.

8. , Parabolic equations on infinite dimensional spaces, Bull. Inst. Math. Acad. Sinica (to appear).

9. E. Nelson, Probability theory and Euclidean field theory, Lecture Notes in Physics, vol. 25, Springer-Verlag, Berlin and New York, 1973.

10. M. A. Piech, A fundamental solution of the parabolic equation on Hilbert space, J. Funct. Anal. 3 (1969), 85-114.

11. Parabolic equations associated with the number operator, Trans. Amer. Math. Soc. 194 (1974), 213-222.

12. The Ornstein-Uhlenbeck semigroup in an infinite dimensional $L_{2}$ setting, J. Funct. Anal. 18 (1975), 271-285.

13. I. E. Segal, Tensor algebras over Hilbert spaces, Trans. Amer. Math. Soc. 81 (1956), 106-134.

Mathematics Department, National Cheng-Kung University, Tainan, Tarwan 700 